\title{
Factors influencing mitotic yields from suspension cultures of normal and acute leukaemic bone
}

\section{marrows}

\author{
SYLVIA D. LAWLER, BRENDA M. SUMMERSGILL, AND J. P. LEWIS1
}

From the Department of Cytogentics and Immunology, Royal Marsden Hospital and Institute of Cancer Research, London and Surrey, and the Department of Internal Medicine, ${ }^{1}$ Section of Haematology and Oncology, University of California School of Medicine, Davis, California, USA

SYNOPSIS Cytogenetic studies of bone marrow are often hampered by a paucity of dividing cells. In an attempt to improve mitotic yields we have tried to promote mitotic activity in suspension cultures of bone marrow from both normal and leukaemic subjects. Enrichment of the media always increases yield and the addition of bone marrow stimulating factor (BMSF) may do so. The best prospect for improving the yield of mitoses in acute myeloid leukaemia is to culture the marrow in an enriched medium with BMSF.

This study arose from a desire to improve the mitotic yields from leukaemic marrows submitted for cytogenetic analysis. We therefore decided to investigate the effect of culture conditions and the addition of stimulating factors to bone marrow suspension cultures set up for chromosomal analysis. The stimulating factors were prepared in the same way as colony stimulating factor (CSF) used to promote growth of granulocytic bone marrow cells in soft agar (Chervenick and Boggs, 1970).

\section{Material and methods}

BONE MARROW SAMPLES

The bone marrow samples were obtained from patients at the Royal Marsden Hospital, Surrey, who required cytological assessment of their bone marrow.

For the purposes of this paper normal marrows were defined as those from non-leukaemic patients. In the majority of cases these patients were haematologically normal, but two showed marrow infiltration, one with melanoma and one with lymphoma. However, the chromosomes of the cells in division appeared normal. Another patient had a leukaemoid reaction, and a fourth aspirate showed abnormal cytological features of the erythroid series but granulopoiesis was normal. The remaining 43 samples classified as normal showed normal haema-

Received for publication 6 November 1975 topoiesis. The study also included bone marrow samples from 26 patients with untreated acute myeloid leukaemia (AML).

A bone marrow sample from a patient with red cell aplasia and blood samples showing high leucocyte counts from two patients with Philadelphia ( $\mathrm{Ph}$ ') chromosome positive chronic myeloid leukaemia (CML) were also investigated but are not included in the tables.

PREPARATION OF BONE MARROW STIMULATING FACTORS

The stimulating factor was prepared according to the method of Chervenick and Boggs (1970) for production of CSF. The leucocytes, $1.5 \times 10^{9} \mathrm{cell} / \mathrm{l}$, were incubated for 10 days in McCoy's $5 \mathrm{~A}$ at $37^{\circ} \mathrm{C}$ in $5 \% \mathrm{CO}_{2}$ in air as it is essential to maintain an acid pH. After incubation the cells were spun down at $200 \mathrm{~g}$ and the supernatant bone marrow stimulating factor (BMSF) was removed and stored at $-20^{\circ} \mathrm{C}$ until used. Batches of factor (BMSF-N) were produced from 15 normal individuals and from three patients with $A M L, C M L$, and chronic myelomonocytic leukaemia (BMSF-L).

\section{PREPARATION OF BONE MARROW CULTURES}

The bone marrow cells which had been aspirated into heparinized balanced salt solution were spun down and resuspended in a small volume of McCoy's 5A medium. The suspension was then thoroughly mixed before being equally distributed among the 
containers used in a particular experiment. The culture medium consisted of McCoy's 5A with added glutamine and antibiotics with $15 \%$ calf serum. The total volume of each culture was $4 \mathrm{ml}$. The standard concentration of BMSF was $20 \%$.

HARVESTING OF CULTURES, PREPARATION OF SLIDES, AND ANALYSIS OF DATA

Colchicine $(20 \mu \mathrm{g} / \mathrm{ml})$ was added to the cultures two hours before the time chosen for termination. The material was fixed in methanol-acetic acid (3:1), and slides were made from suspensions in $60 \%$ acetic acid. All the material from each experiment was evenly distributed on glass slides from which the total number of metaphases in each experiment was determined.

\section{Results}

CHOICE OF CULTURE MEDIA

Preliminary experiments were done in order to determine whether the composition of the medium or time in culture affected the mitotic yields in bone marrow cultures. In the standard so-called 'direct' preparation for cytogenetic analysis the bone marrow is incubated for 2 hours at $37^{\circ} \mathrm{C}$ in buffered salt solution (Tjio and Whang, 1962). With both normal and leukaemic marrows in cultures incubated for 2 or 24 hours the yield of mitoses was increased approximately threefold when McCoy's 5A medium or Ham's medium with glutamine was used instead of the balanced salt solution. Since in the preparation of bone marrow stimulating factors the leucocytes were incubated with McCoy's 5A this medium was used in all the experiments.

\section{EFFECT OF INCUBATION TIME}

As seen in table $I$, there appears to be a different type of response between normal and AML marrow when yields at 2 and 24 hours in the enriched medium are compared. It is characteristic of normal marrow that the yield of mitoses is greater after 24 hours' incubation than after 2 hours. On the other hand, although the samples of AML marrows did not

\begin{tabular}{lllll}
\hline & \multicolumn{2}{l}{$\begin{array}{l}\text { Ratio of } \\
\text { Number of mitoses at 24 } h\end{array}$} \\
\cline { 2 - 5 } & \multicolumn{2}{l}{ Number of mitoses at $2 \mathrm{~h}$} \\
\cline { 2 - 5 } & $<0.7$ & $0.7-1 \cdot 3$ & $1.4-3.9$ & $>3.9$ \\
\hline Number of normal cases & $1^{1}$ & 0 & 9 & 21 \\
Number of AML cases & 10 & 1 & 3 & 3 \\
\hline
\end{tabular}

Table I Comparison of number of mitoses at 24 hours and at 2 hours

${ }^{1}$ Case with leukaemoid reaction behave uniformly, 10 out of the 17 examined showed a decrease in mitotic yield after 24 hours as compared to 2 hours.

\section{EFFECT OF BMSF-N}

The effect of incubating normal marrow with BMSF-N for 2 hours is not uniform. The number of mitoses may be increased $(12 / 31)$ or decreased $(8 / 31)^{\text {s }}$ (table II). The response of marrows from patientș

\begin{tabular}{lllllll}
\hline & $\begin{array}{l}\text { Ratio of } \\
\text { Number of mitoses at } 2\end{array}$ & $h+B M S F-N$ \\
\cline { 2 - 6 } & \multicolumn{2}{l}{\begin{tabular}{l} 
Number of mitoses at \\
\cline { 2 - 6 }
\end{tabular}} & $h$ without & $B M S F-N$ \\
\cline { 2 - 6 } & $<0.7$ & $0.7-1.3$ & $1.4-3.9$ & $>3.9$ \\
\hline Number of normal cases & 8 & 11 & 6 & 6 & 0 \\
Number of AML cases & 6 & 5 & 13 & 2 & 0 \\
\hline
\end{tabular}

Table II Comparison of number of mitoses at 2 hours with and without BMSF-N

\begin{tabular}{|c|c|c|c|c|}
\hline & \multicolumn{4}{|c|}{$\begin{array}{l}\text { Ratio of } \\
\text { Number of mitoses at } 24 h+B M S F-N\end{array}$} \\
\hline & \multicolumn{4}{|c|}{ Number of mitoses at $24 h$ without BMSF- } \\
\hline & $<0.7$ & $0 \cdot 7-1 \cdot 3$ & $1 \cdot 4-3 \cdot 9$ & $>3 \cdot 9$ \\
\hline Number of normal cases & 9 & 9 & 19 & 9 \\
\hline Number of AML cases & 6 & 2 & 8 & 1 \\
\hline
\end{tabular}

Table III Comparison of number of mitoses at 24 hours with and without BMSF-N

with AML to BMSF-N also varied but the number? of mitoses was increased in more than half the cases $(15 / 26)$.

Comparing the number of mitoses at 24 hours with and without BMSF-N (table III), 28 out of 460 . normal marrows and nine out of 17 AML marrowş showed an increased yield.

INDIVIDUAL VARIATION IN BMSF-N AND EFFECT OF CONCENTRATION

The degree to which BMSF-N stimulated mitoses̃ varied. In general, donors whose BMSF-N was highlyn active on one occasion yielded highly active factors on repeated testing. We also studied the ability of various concentrations of BMSF-N to stimulate mitoses of normal marrow. Ten per cent and $75 \%$ BMSF-N were found to be less stimulatory than either $20 \%$ or $40 \%$ BMSF-N. The optimal con:centration was $40 \%$ : however, for reasons of economy we used $20 \%$ BMSF-N for all other experiments.

STIMULATING FACTORS MADE FROM LEUKAEMIC BLOOD (BMSF-L) The preparations of BMSF-L appeared to be active and the same patterns of response followed as witp 
BMSF-N when they were applied to both normal and leukaemic bone marrow samples. When a stimulatory result was obtained with BMSF-N the increase in mitotic activity obtained with BMSF-L was usually less.

\section{EVIDENCE THAT BMSF-N STIMULATES \\ MYELOID CELLS}

BMSF-N does not stimulate mitoses in normal peripheral blood lymphocyte cultures.

In blood cultures from two $\mathrm{Ph}^{\prime}$ positive patients the mitoses obtained with and without BMSF-N were $\mathrm{Ph}^{\prime}$ positive. No $\mathrm{Ph}^{\prime}$ negative mitoses were seen in the cultures with BMSF-N. In blood from these patients, cultured with phytohaemagglutinin, both $\mathrm{Ph}^{\prime}$ positive and negative cells were observed.

One of the patients with AML had an abnormal chromosome, $16 \mathrm{q}+$, in some of the bone marrow metaphases. This $16 q+$ chromosome was also seen in divisions obtained after the addition of BMSF-N. The patient never went into remission and the $16 \mathrm{q}+$ was still present in the bone marrow sample incubated with and without BMSF-N after treatment.

Additional supportive data come from a case of red cell aplasia. The yield of mitoses at 2 hours and at 24 hours in cultures without BMSF-N was 117 and 347 respectively, indicating a normal marrow response pattern. After addition of BMSF-N the number of mitoses was increased, the ratio (number of mitoses + BMSF-N/number of mitoses in control) being 3.9 at 2 hours and 1.7 at 24 hours.

\section{Discussion}

Cytogenetic analyses of bone marrow cells are often restricted by an inadequate yield of dividing cells even in samples of adequate cellularity. This is surprising in view of the fact that the marrow is in cycle, up to $34 \%$ of the cell population being capable of dividing, with a generation time of between one and two days (Wintrobe et al, 1974) Even though the generation time of myeloid cells in AML may be longer than normal, this is compensated for by a very high proportion of cells having division potential (Clarkson et al, 1967; Clarkson et al, 1970). Rather than proposing an inadequate mitotic rate in the marrow as the cause of cytogenetic failure, it is pertinent to consider whether the deficiency of mitoses is due to the non-physiological conditions used in processing the marrow. It is therefore relevant that we have observed an increased mitotic yield when using enriched media.

Even using the enriched medium the number of mitoses observed in normal bone marrows at 24 hours was greater than at 2 hours. This appears to be the typical normal response. On the other hand, marrows from untreated patients with AML usually yield fewer mitoses at 24 hours than at 2 hours.

Normal human bone marrow contains cells which proliferate and mature in semisolid media under the influence of CSF, producing clusters and colonies ranging in size from 3 to 300 cells. Some acute myeloid leukaemic marrow also contains such cells, although the frequency of the large colonies is less and the incidence of clusters often increased (Metcalf, 1973).

CSF has been described as a glycoprotein with molecular weight of 45000 daltons. Others have suggested that CSF is composed of subunits of 15-20 000 daltons and that by combination the MW might vary from 15000 to 70000 daltons. CSF is species non-specific, produced by monocytes, and is thought to participate in the regulation of normal granulopoiesis in vivo as well as serving as a stimulating factor in semisolid media (Metcalf, 1973). These factors may be produced in a variety of ways.

In our studies we used the technique of Chervenick and Boggs (1970) to produce BMSF, and we have found the supernatant from these cultures to be capable of stimulating mitoses in suspension cultures in both normal and leukaemic marrows. Since we have not proved our material to be identical with CSF, we have chosen to call it BMSF. Further studies may show that both factors are identical.

It is possible that the effects of BMSF-N are due to a speeding up of mitotic rate rather than recruitment of dormant cells into the mitotic pool. It appears that BMSF-N can cause either an increase or decrease in mitotic yield from normal marrows (tables II and III), and alteration of mitotic cycle time could explain both effects. The action of BMSF-N on AML marrows was also variable but more often stimulatory than inhibitory.

Some preliminary experiments on factors made from patients with chronic and acute myeloid leukaemia indicate that BMSF can be produced by the leucocytes of these patients. The success may simply mirror the state of the monocyte population. Goldman et al (1974) failed to make CSF from the cells of patients with Philadelphia positive CML chromosome and high leucocyte counts but succeeded when the counts were normal after treatment.

BMSF-N does not act as a mitotic stimulant of normal lymphocytes. By using abnormal chromosomes present in cells of myeloid origin as markers, we have been able to show that BMSF-N does stimulate cells of the myeloid series in suspension cultures. Whether the factor also stimulates the erythrocytic and megakaryocytic series remains to be seen. 
Our study shows that mitotic yields in normal marrows may be increased by enriching the media and by using 24-hour incubation times. The addition of BMSF-N to 2-hour cultures in an enriched medium appears to be the best way of improving the prospects for cytogenetic analysis in AML.

We wish to thank the staff of the Department of Clinical Pathology at the Royal Marsden Hospital for their cooperation in supplying us with the bone marrows used in these studies, and Dr B. R. Reeves for a constructive criticism of the manuscript.

\section{References}

Chervenick, P. A. and Boggs, D. R. (1970). Bone marrow colonies: stimulation in vitro by supernatant from incubated human blood cells. Science, 169, 691-692.

Clarkson, B., Fried, J., Strife, A., Sakai, Y., Ota, K., and
Ohkita, T. (1970). Studies of cellular proliferation in human leukemia. III. Behavicr of leukemic cells in three adults with acute leukemia given continuous infusions of ${ }^{3} \mathrm{H}$-thymidine for 8 or 10 days. Cancer (Philad.), 25, 1237-1260.

Clarkson, B., Ohkita, T., Ota, K., and Fried, J. (1967).믐 Studies of cellular proliferation in human leukemia. I. $\bar{\sigma}$ Estimation of growth rates of leukemic and normal hematopoietic cells in two adults with acute leukemia given single injections of tritiated thymidine. J. clin. Invest. 46, 506-529.

Goldman, J. M., Th'ng, K. H., and Lowenthal, R. M. (1974).. In vitro colony forming cells and colony stimulating factor $\overrightarrow{\vec{Z}}$ in chronic granulocytic leukaemia. Brit.J. Cancer, 30, 1-12.

Metcalf, D. (1973). Regulation of granulocyte and monocyte-? macrophage proliferation by colony stimulating factor (CSF): a review. Exp. Hemat., 1, 185-201.

Tjio, J. H. and Whang, J. (1962). Chromosome preparations of bone marrow cells without prior in vitro culture or $v$ in vivo colchicine administration. Stain Technol., 37, 17-20.

Wintrobe, M. M., et al. (1974). Clinical Hematology, 7thw edition. Lea and Febiger, Philadelphia. 